\title{
Evaluation of Four Models for Predicting Thermal Sensation in Chinese Residential Kitchen
}

\author{
Xiaojie Zhou ${ }^{1}$, Sumei Liu ${ }^{1}$, Xuan Liu ${ }^{1}$, Xiaorui Lin $^{2}$, Ke Qing ${ }^{2}$, Weizhen Zhang ${ }^{2}$, Jian Li $^{3}$, Jiankai Dong ${ }^{3}$, Dayi Lai ${ }^{4}{ }^{*}$ and \\ Qingyan $\mathrm{Chen}^{5}$ \\ ${ }^{1}$ School of Environmental Science and Engineering, Tianjin University, Tianjin, 300072, China \\ ${ }^{2}$ Vanke Real Estate Development Co. Ltd, Changsha Vanke, Hunan Province, 410000, China \\ ${ }^{3}$ School of Architecture, Harbin Institute of Technology, Heilongiiang Province, 150006, China \\ ${ }^{4}$ Department of Architecture, School of Design, Shanghai Jiao Tong University, Shanghai, 200240, China \\ ${ }^{5}$ School of Mechanical Engineering, Purdue University, West Lafayette, IN 47905, USA
}

\begin{abstract}
Thermal environment in residential kitchen in China is transient and non-uniform and with strong radiation asymmetry from gas stove. Due to the complexity of kitchen thermal environment, it is not sure if previous thermal comfort models can accurately predict the thermal comfort in residential kitchens. In order to evaluate if existing thermal comfort models can be applied for Chinese kitchens, this investigation conducted human subject tests for 20 cooks when preparing dishes in a kitchen. The study measured skin temperatures of the cooks and environmental parameters and used questionnaires to obtain their thermal sensation votes at the same time. The actual thermal sensation votes were compared with the predicted ones by four thermal comfort models: predicted mean vote (PMV) model, dynamic thermal sensation (DTS) model, the University of California at Berkeley (UCB) model, and the transient outdoor thermal comfort model from Lai et al. The results showed that all the models could predict the trend of the thermal sensations but with errors. The PMV model overpredicted the thermal sensations. The UCB and Lai's models showed a slower change in thermal sensation votes (TSV) after turning on the stove. The DTS model was more accurate than the others in predicting the mean thermal sensation, but with a large variation in predicting individual thermal sensation votes. A better thermal comfort model should be developed for Chinese residential kitchens.
\end{abstract}

\section{Introduction}

Cooking is a very important daily activity for a family. The average time for a family member spent in kitchen in China is about 3.6 hours per day $[1,2]$. Thus, it is important to have an acceptable thermal environment in Chinese kitchen. The thermal environment is related to cooking activities, such as frying, stir-frying, stew, steaming, etc. The use of high power gas stove in Chinese residential kitchen generates a lot of heat, which would deteriorate the thermal environment [3]. The radiant heat from the stove makes the thermal environment particularly hot in summer [4]. To evaluate thermal comfort level in Chinese kitchen requires an appropriate model.

The most frequently used thermal comfort model for kitchen is the predicted mean vote (PMV) index $[5,6]$ that could further calculate predicted percentage dissatisfied (PPD). For example, Rahmillah et al. [7] can predict thermal comfort in conventional kitchen with gas stoves with PMV and PPD. However, Simone and Olesen [8] investigated the thermal environment in more than 100 commercial kitchens in the United States and found that in many cases the measured conditions were outside the prediction range of the PMV due to the high thermal radiation from the appliances in the cooking zone. Because PMV index is intended for buildings where its thermal environment is steady and uniform with sedentary or near-sedentary activity levels, it may not be suitable to use PMV to assess thermal sensation for cooking activities in kitchens.

Since the thermal environment in a residential kitchen in China is transient and non-uniform due to strong radiation asymmetry from gas stove, PMV may not accurately evaluate the thermal comfort level. Thus, this study identified other three thermal comfort models that could account for the transient and non-uniform situations. These three models are (1) the dynamic thermal sensation model (DTS) from Fiala [9], which was based on regression analysis of thermal sensation votes from experiment in a climate chamber and human physiological responses (skin temperature, hypothalamus temperature, rate of change in skin temperature) calculated from a multi-segment human heat transfer model; (2) the University of California at Berkeley (UCB) model [10], which was based on large-scale

Corresponding author: dayi lai@,situ.edu.cn 
experimental tests of local and overall thermal sensations and thermal comfort; and (3) the dynamic outdoor thermal comfort model from Lai et al. [11], which used thermal load, mean skin temperature, and the change rate of the mean skin temperature as the predictor variables for thermal sensation.

To evaluate if the above four models (PMV, DTS, UCB, and Lai's) could be used to predict thermal comfort in Chinese residential kitchens, this study collected data from 20 cooks through human subject tests and compared the predicted thermal comfort level with the actual thermal sensation votes.

\section{Methods}

Our study used human subject tests to obtain actual thermal sensation votes in a Chinese kitchen mockup as shown in Fig. 1(a). The subjects were 20 cooks from a cooking school. The study measured their skin temperatures, kitchen thermal environmental parameters and clothing levels of the cooks under a standardized cooking activity (boiling water in a standing position, with a metabolic rate considered to be 1.3 met). The cooks were asked to rate their thermal sensation levels during the cooking process. The thermal environmental parameters, the clothing insulation, and the metabolic rate were used to calculate the thermal comfort levels of the cooks by the four identified comfort models. The actual thermal sensations were compared with the predicted thermal comfort level in order to evaluate the comfort model. This section describes the experimental procedure and the four comfort models.
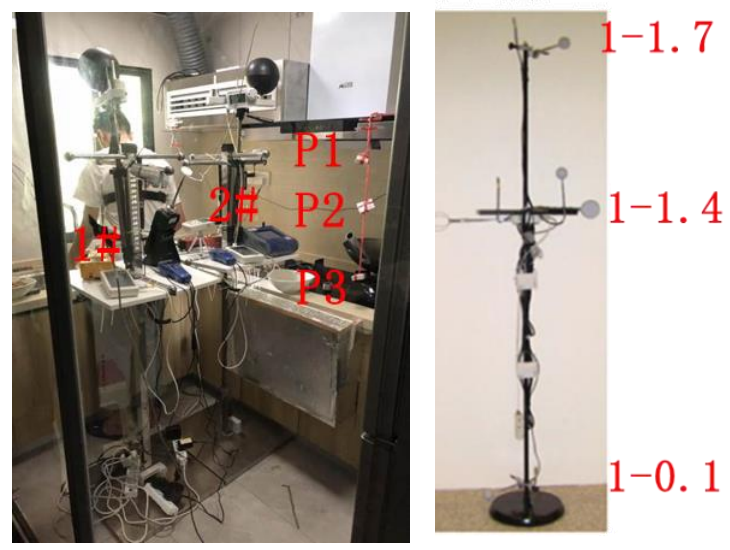

Fig. 1. (a) The kitchen mockup and (b) Environmental test instrument.

\subsection{Experimental procedures}

The human subject tests were conducted in a kitchen in Changsha, China with an exterior window and an interior door and the kitchen was $2.9 \mathrm{~m}$ long $\times 1.85 \mathrm{~m}$ wide $\times 2.3 \mathrm{~m}$ high. Twenty subjects, 10 males and 10 females with an average age of 25 (standard deviation $=$ 3.4), participated in the tests. The subjects were cooks from a cooking school. Each subject was asked to engage a simulated standardized cooking activity (boiling water in a standing position) to ensure that the operation of each subject and their metabolic rate were nearly the same. Each test took about one hour.

The experimental procedure was as follows: Before each test, the exterior window and interior door were opened and a portable fan in the kitchen and the exhaust hood were turned on for about 20 minutes to ensure the kitchen started with a non-conditioned state as the situation in most Chinese residential kitchens. After that, the window and door in the kitchen were closed for 10 minutes. During the 30 minutes, we asked the subject to stay in a preparation room with an ambient temperature close to $26^{\circ} \mathrm{C}$ to achieve a neutral thermal state. During this period, the subject was briefed about the experimental procedure and was taped with the wireless button thermometers on their skin surface. Then the subject went to the kitchen. After a 5-minute rest period, the subject turned on the stove and the exhaust hood, and began the boiling water activity.

During the boiling water activity, we conducted subjective questionnaire survey of the subject by asking cook's thermal sensation vote (TSV) based on ASHRAE 7-point scale and objective on-site measurements of the indoor/outdoor air temperature, relative humidity and cook's skin temperature $\left(T_{s k}\right)$. Fig. 1(b) shows the air temperature was measured at different heights. For more information about the experiment, please see our companion paper from Liu et al. [12].

\subsection{Thermal confort models}

\subsubsection{Predicted mean vote (PMV) model}

PMV model was developed by Fanger [5] using data from 1393 American and Danish subjects in climate chamber. The mean thermal sensation was regressed against metabolic rate $\left(M, \mathrm{~W} / \mathrm{m}^{2}\right)$ and thermal load $(T L$, $\mathrm{W} / \mathrm{m}^{2}$ ) calculated by six environmental and human parameters (air temperature, humidity, air velocity, mean radiant temperature, clothing insulation, and metabolism rate). PMV is linked to $T L$ by:

$$
\begin{gathered}
P M V=[0.303 \cdot \exp (-0.0368 M)+0.028] \cdot T L \\
T L=(M-W)-\left(C+R+E_{s k}+C_{r e s}+E_{r e s}\right)
\end{gathered}
$$

where $M$ is metabolism rate $\left(\mathrm{W} / \mathrm{m}^{2}\right), W$ rate of mechanical work $\left(\mathrm{W} / \mathrm{m}^{2}\right), C$ rate of convective heat loss $\left(\mathrm{W} / \mathrm{m}^{2}\right), R$ rate of radiative heat loss $\left(\mathrm{W} / \mathrm{m}^{2}\right), E_{s k}$ rate of evaporative heat loss from the skin $\left(\mathrm{W} / \mathrm{m}^{2}\right)$, and $C_{\text {res }}$ and $E_{\text {res }}$ rates of convective and evaporative heat loss, respectively, from respiration $\left(\mathrm{W} / \mathrm{m}^{2}\right)$.

\subsubsection{Dynamic thermal sensation (DTS) model}

The DTS model was mainly based on the regression of a large number of independent experimental data. The experimental data used in the model was obtained from a large ambient temperature range $\left(5-50^{\circ} \mathrm{C}\right)$. The DTS transient thermal sensation model was expressed as: 


$$
\begin{aligned}
D T S= & 3 \cdot \tanh \left[a \cdot \Delta T_{s k, m}+F_{2}+\left(0.11 \cdot \frac{d T_{s k, m}^{(-)}}{d t}\right.\right. \\
& \left.\left.+1.19 \cdot\left(\frac{d T_{s k, m}^{(+)}}{d t}\right)_{\max } \cdot e^{-0.681 t}\right) \cdot \frac{1}{1+F_{2}}\right]
\end{aligned}
$$

where $T_{s k, m}\left({ }^{\circ} \mathrm{C}\right)$ and $T_{h y}\left({ }^{\circ} \mathrm{C}\right)$ are the mean skin temperature and the hypothalamus temperature, respectively, while $\Delta T_{s k, m}\left({ }^{\circ} \mathrm{C}\right)$ and $\Delta T_{h y}\left({ }^{\circ} \mathrm{C}\right)$ the differences between the actual and neutral $T_{s k, m}$ and $T_{h v}$ respectively; $d T_{s k, m}(-) / d t$ negative rates of change of the mean skin temperature as a dynamic signal influencing regulatory responses against cold, i.e. shivering and vasoconstriction; $\left(d T_{s k, m}(+) / d t\right)_{\max }$ positive changing rate of mean skin temperature as a dynamic signal influencing regulatory responses against hot, i.e. sweating and vasodilation; and $F_{2}$ stands for the effect of core temperature on thermal sensation as a multiple function of the temperature error signals of $\Delta T_{s k, m}$ and $\Delta T_{h y}$ :

$$
F_{2}=7.94 \cdot \exp \cdot\left(\frac{-0.902}{\Delta T_{h y}+0.4}+\frac{7.612}{\Delta T_{s k, m}-4}\right)
$$

Because of the short experiment time, we did not measure the hypothalamus temperature, the $\Delta T_{h y}$ was assumed to be $0{ }^{\circ} \mathrm{C}$.

\subsubsection{University of California at Berkeley (UCB) model}

The original intention developing UCB model was to study thermal comfort in a non-uniform environment. It was based on the regression of a large number of experimental data from local cooling/heating of the skin of different segments in experimental chamber. The local thermal sensation model from UCB has a static term that is based on the deviation of the local skin temperature from its set-point and a dynamic term based on the time derivative of the skin and core temperatures. It can be represented by:

$$
\begin{aligned}
T S_{\text {local }, i}= & 4 \cdot\left(\frac{2}{1+\exp \cdot\left[-C 1 \Delta T_{s k, i}-K 1\left(\Delta T_{s k, i}-\Delta T_{s k, m}\right)\right]}\right. \\
& -1)+C 2 \cdot \frac{d T_{s k, i}}{d t}+C 3 \cdot \frac{d T_{c r}}{d t}
\end{aligned}
$$

where $T S_{\text {local }, i}$ is local thermal sensation (based on ASHRAE 9-point scale); $T_{s k, i}$ local skin temperature $\left({ }^{\circ} \mathrm{C}\right)$; and $\Delta T_{s k, i}$ difference between actual and neutral local skin temperature $\left({ }^{\circ} \mathrm{C}\right), T_{s k, m}$ mean skin temperature $\left({ }^{\circ} \mathrm{C}\right)$, and $\Delta T_{s k, m}$ difference between actual and neutral mean skin temperature $\left({ }^{\circ} \mathrm{C}\right) ; C 1$ and $K$ coefficients that varies for different body parts; and $C 2$ and $C 3$ regression coefficients at the skin and core nodes, respectively.

The overall thermal sensation is just the weighted average of the local thermal sensations:

$$
T S_{\text {overall }}=\frac{\sum \text { weight }_{i} \cdot T S_{\text {local }, i}}{\sum \text { weight }_{i}}
$$

The weights have different values for various body segments. Additionally, when the body is at a different thermal state (for example, when the body is warm or cold), the values of the weighting coefficients may change.

\subsubsection{Transient outdoor thermal comfort model (Lai's model)}

The Lai's model was mainly based on the regression of a large number of experimental data obtained under different outdoor meteorological environments. Thus, the model is for evaluating outdoor thermal comfort. Since outdoor conditions are transient and sometimes nonuniform, the model may be used for kitchen environment. Lai's model can be expressed by:

$$
T S_{\text {overall }}=3\left(1-\frac{2}{1+\exp \left(B_{1} \cdot T L+B_{2} \cdot \Delta T_{s k, m}+B_{3} \cdot d T_{s k, m} / d t\right.}\right)
$$

Where $\Delta T_{s k, m}\left({ }^{\circ} \mathrm{C}\right)$ is the difference between actual and neutral $T_{s k, m} ; B_{1}, B_{2}$, and $B_{3}$ regression coefficients for $T L, \Delta T_{s k, m}$, and $d T_{s k, m} / d t$, respectively (different seasons have different regression coefficients); and $T L$ calculated thermal load from Equation (2).

\section{Results}

This section first introduced the results of all the 20 tests. Then compared the actual TSV with the predicted thermal sensations by the four thermal sensation models for two typical subjects with different gender, the mean actual and predicted thermal sensation, and for individual votes.

\subsection{Air temperature and relative humidity variation in the kitchen and actual TSV from the tested subjects}

The results of environmental parameters obtained from 20 experiments were statistically analyzed, and the distribution of 20 sets of data was given by box plot. Figure 2 showed the change of air temperature and relative humidity at the breathing area (2-1.4) during the experiment for all the 20 tests. The air temperature difference between the subjects was less than $4{ }^{\circ} \mathrm{C}$, and the relative humidity was less than $15 \%$. The temperature and relative humidity variation range of the remaining eight air measuring points is consistent with the trend of measuring point at the breathing area (21.4).

After processing the data, the median value of this set of test data was almost equal to the average value, which 
also shows that the experimental data was symmetrically distributed. Figure 3 showed the mean value of the air temperature and relative humidity results of different nine points (see Figure.1) during the experiment. The indoor air temperature began to rise when the subject turned on the stove and it continuously to rise with the cooking process. The air temperature at the "P3" position was the highest because it was most close to the stove as shown in Figure 1. It rose by $11.7^{\circ} \mathrm{C}$ compared from its initial temperature at the beginning of the cooking.

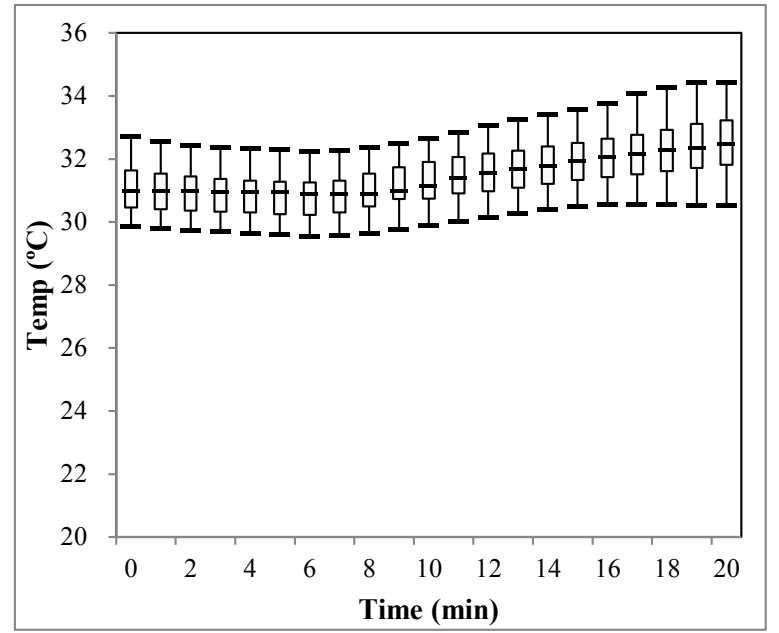

(a)

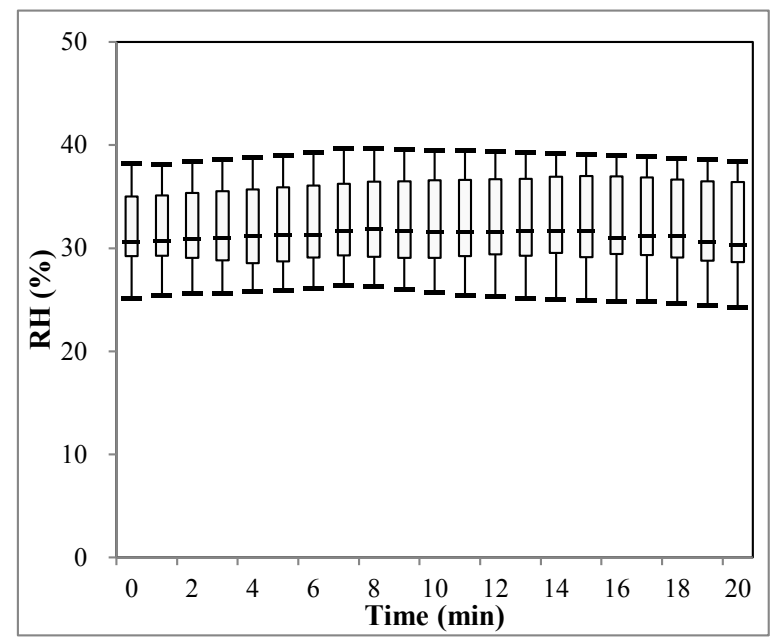

(b)

Fig. 2. Box plot of (a) the air temperature variation, (b) the relative humidity variation at the breathing area during the tests. The horizontal lines represents the minimum, 25th, 50th (the median), 75th percentiles, and maximum.

In addition, the air temperature stratification existed in the kitchen. The vertical temperature difference at the $1 \#$ measuring point (the temperature difference between the measuring point 1-1.7 and 1-0.1) increases from the initial $2.8{ }^{\circ} \mathrm{C}$ to $4.6{ }^{\circ} \mathrm{C}$, and the vertical temperature difference at the $2 \#$ measuring point increases from the initial $3.8{ }^{\circ} \mathrm{C}$ to $6.1{ }^{\circ} \mathrm{C}$. It can be found that the air temperature around the subject was highly non-uniform. The relative humidity did not show significant difference in both $1 \#$ and $2 \#$. While the relative humidity at the stove locations (P1, P2, and P3) began to decrease were when the subject turned on the stove for cooking and it continuously decreased with the cooking process, which was reasonable because of the rise of the air temperature.

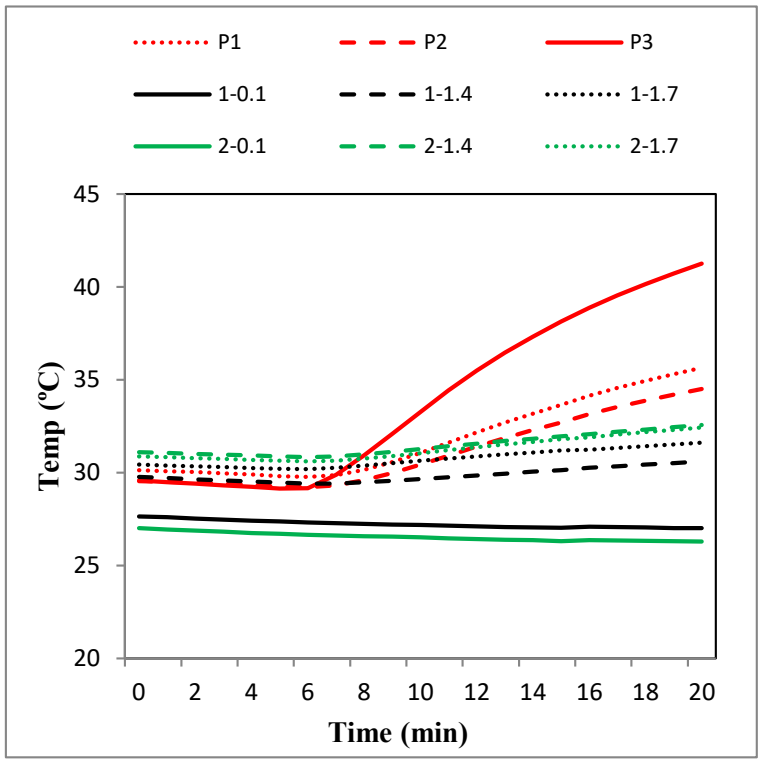

(a)

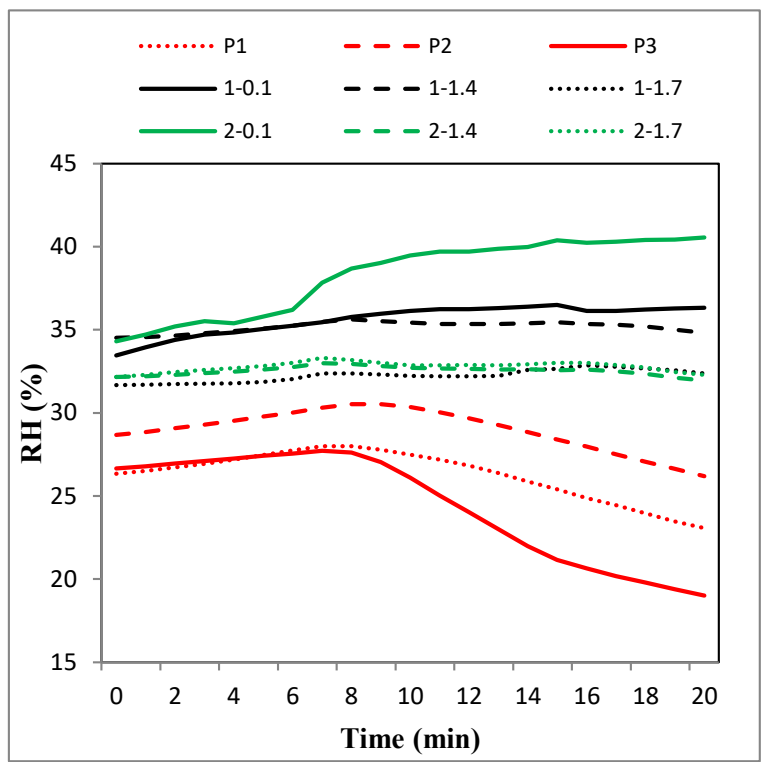

(b)

Fig. 3. Mean value of environmental parameters distribution: (a) indoor air temperature distributions, and (b) indoor relative humidity distributions.

Figure 4 showed the box plot of the actual thermal sensation votes from the 20 subjects over the 20 minute test period. The figure demonstrates individual differences among the 20 subjects with the largest difference near three units under similar air temperature in the kitchen. The thermal sensation of the tested cooks started at near neutral level, but it increased because of radiant heat from the gas stove after five minutes. It should be noted that the subject's thermal sensation vote increased 0.5 units at the moment of opening the stove, while the increase in air temperature occurred 2 minutes after the opening. At around 15 minutes, the median TSV 
almost reached the highest level.

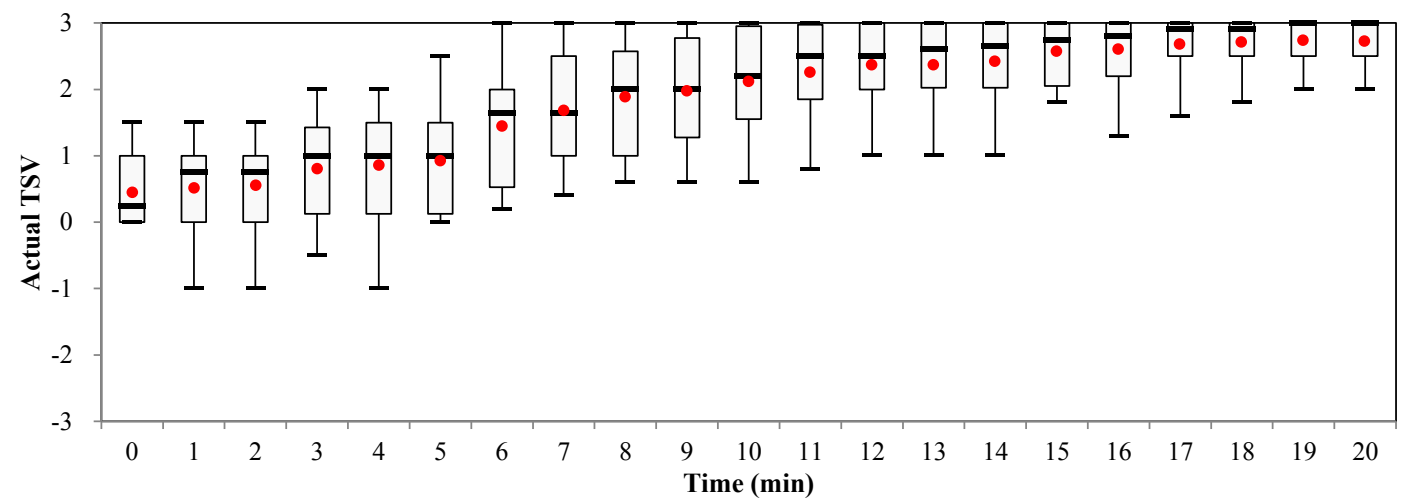

Fig. 4. Box plot of the TSV for the 20 tested subjects during the experiment. The horizontal lines represent the minimum, $25^{\text {th }}, 50^{\text {th }}$ (the median), $75^{\text {th }}$ percentiles, and maximum. The red solid dots represent average TSV.

\subsection{Model evaluation by the thermal sensation votes from typical subjects}

Women's experiences within the domestic kitchen have been documented and recorded, as they are seen as the gatekeepers of that space within the home, while commercial kitchens are still dominated by men. Therefore, this paper also considered the influence of gender on the subjective feelings of the kitchen thermal environment and thermal comfort.

Due to the limited space available for this paper, we evaluated the model by using TSV from two typical subjects (one male and another female). The TSV is similar to the median TSV from all the 20 subjects. Figure 5 compares the predicted TSV obtained by the four models (PMV, DTS, UCB and Lai's) and the actual TSV. Though the predicted TSV had similar trend as the actual TSV, the differences were large.

The TSV predicted by the PMV model were much larger than the actual TSV. The PMV index was intended for buildings with steady and uniform environment and with sedentary or near-sedentary activity levels, while the thermal environment in the kitchen was non-uniform and transient during cooking. The large discrepancy is understandable. Lai's model predicted a larger TSV than the actual TSV at the beginning of the experiment. After turning on the stove, the prediction by Lai's model began to be one unit lower than the actual TSV. This may be due to the thermal load of the human body had mutation process and increased during the cooking progress, so the change rate of the thermal load not considered in Lai's model may also be an important indicator for reflecting the thermal sensation during the kitchen environment .

The UCB model predicted almost the same TSV as the actual one before boiling water. Similar to Lai's model, the UCB model predicted a slower increase of TSV than the actual TSV after the gas stove was on. This may be due to the segmental skin temperatures and their change rates used in the model were insufficient to reflect the change in the thermal sensation. The DTS model was the most accurate one, with the discrepancies less than 0.7 units.

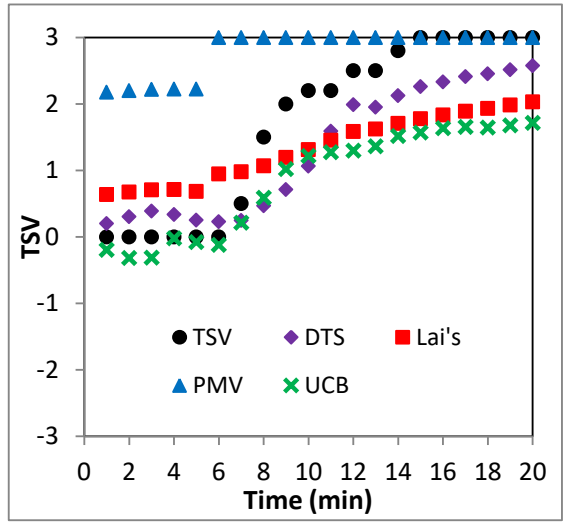

(a)

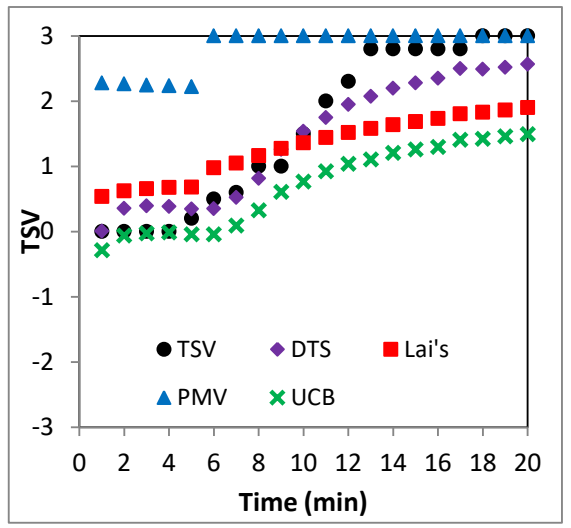

(b)

Fig. 5. Comparison between the actual and predicted thermal sensation by the four models (a) for a male subject and (b) for a female subject.

\subsection{Model evaluation by the mean thermal sensation votes}

A total of 420 thermal sensation votes were given by the 20 subjects. We calculated the mean actual TSV for the 20 subjects at each time interval for the comparison with that predicted by the four thermal comfort models.

Figure 6 shows there were no major differences on the TSV between male and female test subjects. This may 
because the environment was warm-hot during the cooking process in summer kitchen, as results of previous study, there was no difference on thermal comfort between genders in a neutral-warm environment, while the impact of gender differences on thermal comfort in a neutral-cold environment cannot be ignored.

The results also showed the mean TSV from the 10 male and 10 female subjects to be consistent with that from the typical subjects. Again, the PMV model overpredicted the thermal sensation that was found by many researchers. For example, De Dear and Brager [12] found PMV overestimated subjective warmth sensations of people in warm naturally ventilated buildings. The Lai's and UCB model gave a lower TSV after the gas stove was on. The maximum error was about one unit. The DTS model was the most accurate, because the thermal environment of the kitchen cooking process was in line with its scope.

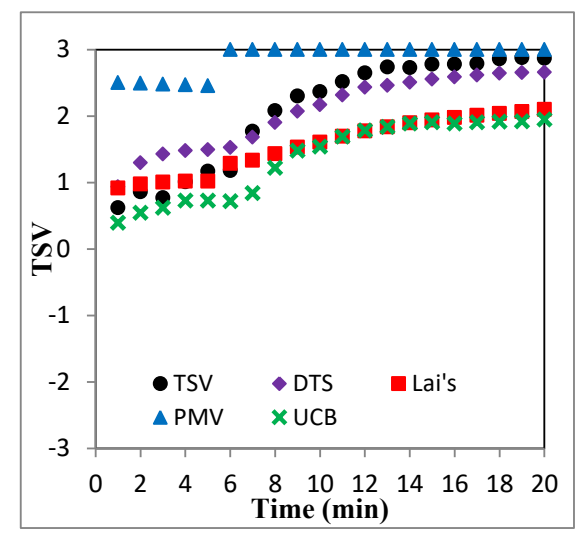

(a)

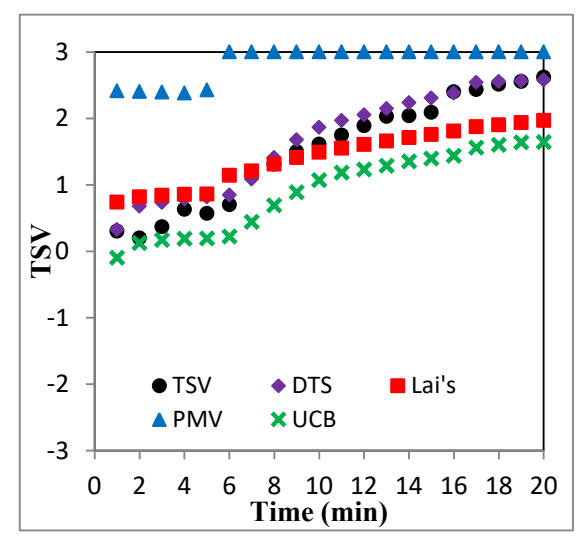

(b)

Fig. 6. Comparison between the actual and predicted mean thermal sensation by the four models (a) for male subjects and (b) for female subjects.

\subsection{Model evaluation for individual thermal sensation votes}

After the analysis of the mean value, we used the scatter plot to assess the accuracy of model predictions for each individual thermal sensation vote, as shown in Figure 7. The results showed that $60.8 \%, 71.1 \%, 78.7 \%$, and $83.9 \%$ of the predicted votes by PMV, UCB, Lai's and
DTS models, respectively, had a difference of less than one unit from the actual TSV. The predicted thermal sensation votes differed from the actual ones. The largest difference was three units for DTS model, two units for UCB model and less than two units for Lai's model. Lai's model showed the smallest variance in the predicted TSV.

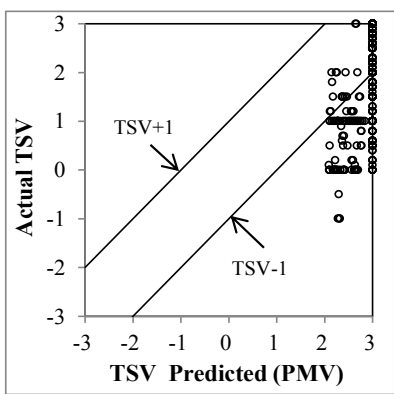

(a)

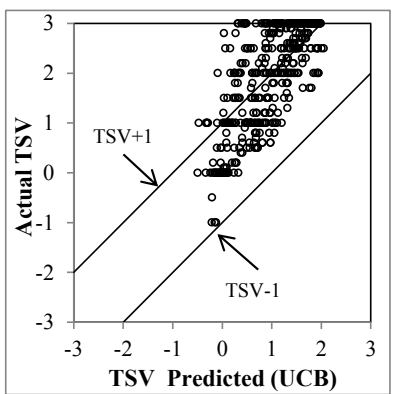

(c)

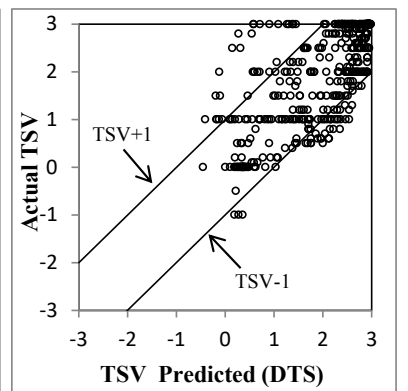

(b)

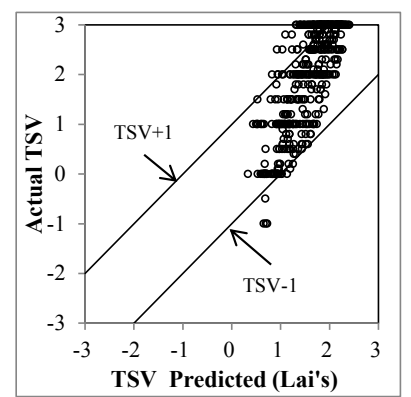

(d)
Fig. 7. Comparison of the actual individual thermal sensation with the predicted one by (a) PMV; (b) DTS; (c) UCB; (d) Lai's model. "TSV+1" and "TSV-1" are the lines where predictions are one unit higher or lower than the actual value.

The first three models were developed based on the data from indoor experiments and the fourth model (Lai's model) based on data from outdoor experiment. The thermal environment in the kitchen was non-uniform and transient due to the strong radiation asymmetry from the gas stove and high activity levels for cooking that may be closer to the outdoor environment for Lai's model. Nevertheless, none of the models seems to give acceptable results. Thus, it is necessary to develop a better model for predicting the thermal comfort in Chinese residential kitchens.

\section{Discussion}

To eliminate uncertainties caused by different cooking habits and different choice of heating levels from gas stove when cooking, we asked the subjects to engage a simulated cooking activity (boiling water in a standing position). However, such simulated water boiling activity may deviate from real cooking activities, and the obtained thermal comfort levels may be different from the actual situation.

This experiment was a field test. The thermal environmental parameters were strongly non-uniform and unsteady. This may cause problems when evaluating the model accuracy, since the thermal environment 
encountered by each subject was different slightly.

The core temperature of the human body is an important physiological parameter that influences thermal comfort. However, the short experiment time, the large inter- and intra-personal variability of the core temperature and the difficulties in measuring it prevented us from studying its impact in this study.

\section{Conclusions}

This investigation used 20 human subject tests to assess four thermal comfort models for use in Chinese residential kitchen.

There were no major differences on the TSV between male and female test subjects. The TSV from the typical subject (median subject) was similar to the mean TSV from the 20 subjects.

The results show that all the models could predict the trend of the thermal sensation but none of the models was sufficiently accurate. The PMV model overpredicted the thermal sensation. The UCB model and Lai's model showed a slower change in TSV after the stove was on. The DTS model was more accurate than the other models, but still predicted a large variation when predicting individual thermal sensation vote.

Given the performance assessment of the above models, a transient and non-uniform thermal comfort model should be developed for Chinese residential kitchen.

\section{Acknowledgements}

The research presented in this paper was partially supported by the National Key R\&D Program from the Ministry of Science and Technology, China, on "Green Buildings and Building Industrialization" through Grant No. 2016YFC0700500 and by the National Natural Science Foundation of China through Grant No. 51678395. We would like to express our gratitude to Jiawei Tang and Tao Zhang from Vanke Real Estate Development Co. Ltd, Changsha Vanke, and Yan Zhou and Yujia Qiu from Xiangya School of Medicine, Central South University, for their help in collecting data for the investigation.

\section{References}

1. C.M. Lai. Building Services Engineering Research and Technology. 26, 157-166 (2005).

2. P. Wei, B. Zhou, M. Tan, F. Li, J. Lu, Z. Dong, G. Wang, Y. Xiao. Procedia Engineering. 205, 20412048 (2017).

3. H. Wang, J. Hu, C. Huang, K. Chen, W. Gu, M. Shi. The 6th International Conference on Indoor Air Quality, Ventilation \& Energy Conservation in Buildings. (IAQVEC Oct. 28-31, Sendai, Japan, 2007)

4. C. Chiang, C. Lai, P. Chou, Y. Li. B\&E. 35, 579585 (2000).

5. PO Fanger. Thermal comfort - analysis and applications in environmental engineering. McGraw-Hill, New York. (1973).

6. ISO EN Standard 7730 (2005): Moderate thermal environments - Determination of the PMV and PPD indices and specification of the conditions for thermal comfort.

7. F. Rahmillah, A. Tumanggor, A. Sari. 2017 IOP Conf. Ser.: Mater. Sci. Eng. 215012033

8. A. Simone, B.W. Olesen, J. L. Stoops, A.W. Watkins. HVAC\&R Research. 19, 1001-1015 (2013).

9. D. Fiala, G. Havenith, P Bröde, B. Kampmann, G. Jendritzky. Int J Biometeorol 56 429-441 (2012).

10. H. Zhang, E. Arens, C. Huizenga, T. Han. Building and Environment 45 399-410 (2010).

11. D. Lai, X. Zhou, Q. Chen. Energy and Buildings 149 16-25 (2017).

12. S. Liu, et al. Proceedings of CLIMA 2019 (2019).

13. R.J. de Dear, G.S. Brager. ASHRAE Transactions 104 (1) 27-49 (1998). 